\title{
Establishing reference intervals for triglyceride-containing lipoprotein subfraction metabolites measured using nuclear magnetic resonance spectroscopy in a UK population
}

\author{
Roshni Joshi' $\mathbb{D}$, Goya Wannamethee ${ }^{2}$, Jorgen Engmann', Tom Gaunt ${ }^{2}$, \\ Deborah A Lawlor ${ }^{3,4,5}$, Jackie Price ${ }^{6}$, Olia Papacosta ${ }^{2}$, Tina Shah', Therese Tillin ${ }^{7}$, \\ Peter Whincup ${ }^{8}$, Nishi Chaturvedi', Mika Kivimaki ${ }^{7}$, Diana Kuh', Meena Kumari ${ }^{9}$, \\ Alun D Hughes', Juan P Casas ${ }^{11,12}$, Steve E Humphries', Aroon D Hingorani' and \\ A Floriaan Schmidt ${ }^{1,13}$; on behalf of the UCLEB Consortium
}

\begin{abstract}
Background: Nuclear magnetic resonance (NMR) spectroscopy allows triglycerides to be subclassified into 14 different classes based on particle size and lipid content. We recently showed that these subfractions have differential associations with cardiovascular disease events. Here we report the distributions and define reference interval ranges for 14 triglyceride-containing lipoprotein subfraction metabolites.

Methods: Lipoprotein subfractions using the Nightingale NMR platform were measured in 9073 participants from four cohort studies contributing to the UCL-Edinburgh-Bristol consortium. The distribution of each metabolite was assessed, and reference interval ranges were calculated for a disease-free population, by sex and age group $(<55$, $55-65,>65$ years), and in a subgroup population of participants with cardiovascular disease or type 2 diabetes. We also determined the distribution across body mass index and smoking status.

Results: The largest reference interval range was observed in the medium very-low density lipoprotein subclass (2.5th 97.5th percentile; 0.08 to $0.68 \mathrm{mmol} / \mathrm{L}$ ). The reference intervals were comparable among male and female participants, with the exception of triglyceride in high-density lipoprotein. Triglyceride subfraction concentrations in very-low density lipoprotein, intermediate-density lipoprotein, low-density lipoprotein and high-density lipoprotein subclasses increased with increasing age and increasing body mass index. Triglyceride subfraction concentrations were significantly higher in ever smokers compared to never smokers, among those with clinical chemistry measured total triglyceride greater than
\end{abstract}

'Institute of Cardiovascular Science, Faculty of Population Health, University College London, London, UK

${ }^{2}$ Department of Primary Care \& Population Health, Faculty of Population Health, University College London, London, UK

${ }^{3} \mathrm{MRC}$ Integrative Epidemiology Unit at the University of Bristol, Bristol, UK

${ }^{4}$ Bristol NIHR Biomedical Research Centre, Bristol, UK

${ }^{5}$ Population Health Science, Bristol Medical School, Bristol, UK

${ }^{6}$ Usher Institute, University of Edinburgh, Edinburgh, UK

${ }^{7}$ Department of Epidemiology and Public Health, University College London, London, UK

${ }^{8}$ Population Health Research Institute, St George's, University of London, London, UK
${ }^{9} \mathrm{MRC}$ Unit for Lifelong Health and Ageing, Institute of Cardiovascular Science, Faculty of Population Health Sciences, University College London, London, UK

${ }^{10}$ Institute for Social and Economic Research, University of Essex, Colchester, UK

"Massachusetts Veterans Epidemiology Research and Information Center (MAVERIC), VA Boston Healthcare, MA, USA

${ }^{12}$ Division of Aging, Department of Medicine, Brigham and Women's

Hospital and Harvard School of Medicine, Boston, MA, USA

${ }^{13}$ Department of Cardiology, Division Heart and Lungs, University

Medical Center Utrecht, Utrecht, the Netherlands

Corresponding author:

Roshni Joshi, 222 Euston Road, London NWI 2DA, UK

Email: Roshni.joshi.16@ucl.ac.uk 
$1.7 \mathrm{mmol} / \mathrm{L}$, and in those with cardiovascular disease, and type 2 diabetes as compared to disease-free subjects.

Conclusion: This is the first study to establish reference interval ranges for 14 triglyceride-containing lipoprotein subfractions in samples from the general population measured using the nuclear magnetic resonance platform. The utility of nuclear magnetic resonance lipid measures may lead to greater insights for the role of triglyceride in cardiovascular disease, emphasizing the importance of appropriate reference interval ranges for future clinical decision making.

\section{Keywords}

Analytes, clinical studies, epidemiology studies, laboratory methods, lipids, nuclear magnetic resonance

Accepted: 14th August 2020

\section{Introduction}

Risk factors for atherosclerotic disease include elevated total cholesterol, LDL-cholesterol (LDL-C) and triglycerides (TGs), and are used in disease risk assessment in clinical care. ${ }^{1}$ Elevated TGs are common among people with metabolic syndrome, obesity and type 2 diabetes $(\mathrm{T} 2 \mathrm{DM}),{ }^{2,3}$ and are associated with an increased risk of cardiovascular disease (CVD). ${ }^{4}$ Population-based reference intervals are used as a tool to define thresholds for clinical decisions. For example, clinical measurement of LDL-C for CVD risk assessment, which together with other measurements such as body mass index (BMI) or systolic blood pressure (SBP) can help to determine if lipid-lowering is indicated. ${ }^{5}$

The high-throughput proton $\left({ }^{1} \mathrm{H}\right)$ serum nuclear magnetic resonance (NMR) metabolomics platform developed by Nightingale provides quantitative information on lipoprotein particle size and lipid content representing multiple metabolic pathways. ${ }^{6-8} \mathrm{NMR}$ measures of lipoproteins are increasingly used in epidemiological and genetic studies, and may provide better insights into biological processes when compared to clinical chemistry measures of $\mathrm{TG}$, which represent the sum of all plasma TG. ${ }^{9}$

The Nightingale NMR metabolomics approach has been used in two recent prospective cohort studies that found evidence to suggest a differential association of total serum TG and TG subfractions with coronary heart disease (CHD). ${ }^{10,11}$ For example, total serum TG association with CHD was OR 1.19 (95\% CI 1.10 to 1.28), TG in the VLDL subfractions was associated with CHD in the range of OR 1.12 to 1.22 , whereas TG in the LDL sub-fractions conveyed a relatively lower risk (OR in the range 1.13 to 1.17 ). ${ }^{11}$ The different associations of the 14 TG-subfractions with CHD highlight the need to extend the standard lipid reference intervals to include lipid lipoprotein subfractions.

In the present study, we aim to define reference intervals for 14 TG-containing lipoprotein subfraction metabolites (TG subfractions) using data from multiple UK-based cohorts from the UCL-Edinburgh-Bristol (UCLEB) consortium.

\section{Methods}

\section{Population study sample}

We sourced data from the UCLEB consortium, including NMR metabolite measures in 9073 participants from four cohort studies: The British Regional Heart Study (BRHS), including male aged 60-79 at assessment in 1998-2000, the Whitehall II Study (WHII), including UK government workers aged 45-69 years at assessment in 1997 to 1999, the Southall and Brent Revisited Study (SABRE), a triethnic study including British men and women from European (SABRE1), South Asian (SABRE2) and African Caribbean (SABRE3) descent, and the Caerphilly Prospective Study (CAPS), including male registered in general practice aged 55-69 at assessment in 1989-1993. The design and data collection for the UCLEB Consortium of longitudinal population studies have been described previously. ${ }^{12}$ Age (years), sex (male/female), smoking (ever/never), BMI, CHD, stroke and T2DM variables were collected at the time of NMR blood sample measurement.

\section{Metabolite quantification}

Using Nightingale NMR metabolomics platform, ${ }^{8}$ high-throughput metabolite quantification of 14 TGcontaining lipoprotein subfractions $(\mathrm{mmol} / \mathrm{L})$ was ascertained in fasting and non-fasting serum samples in all contributing studies. To ensure long-term sample integrity, blood samples were stored and transported at $-80^{\circ} \mathrm{C}$ across all contributing UCLEB studies until NMR quantification in 2014. NMR metabolomics platform has been extensively used in epidemiology and genetics studies, ${ }^{13-15}$ and its 
application reviewed and described in detail elsewhere. ${ }^{6,16}$

\section{Statistical analysis}

We removed individuals based on any event of CHD, stroke or T2DM to include a healthy, 'disease free' population. We first assessed the study-specific distribution of each $14 \mathrm{TG}$ subfraction and then pooled individual participant data form all four cohorts into one dataset. Reference intervals were based on the 2.5th and 97.5th percentiles stratified by age and sex. Age group bands were calculated as $<55$ years, 5565 years and $>65$ years. The influence of fasting, age, smoking and BMI on the subfraction distributions was assessed statistically and graphically using 'generalised linear model' (GAM) curves, Kolmogorov-Smirnov (KS) test and using box plots. Reference intervals were additionally calculated in the following groups; (1) participants with CVD (defined as occurrence of either CHD or stroke), (2) participants with T2DM, (3) participants with clinical chemistry total TG greater, or less than $1.7 \mathrm{mmol} / \mathrm{L}$ and (4) TG measured in the fasting and non-fasting state.

\section{Results}

A total of 9073 individuals were included in the main healthy, free of CVD and T2DM study sample, of which $5574(62.8 \%)$ were male (median age 61.7 years, IQR 52.0, 67.6) who had median BMI of 26.0 (IQR 23.9, 28.4) $\mathrm{kg} / \mathrm{m}^{2}$ and $3027(54.2 \%$ ) were current or ex-smokers (i.e. ever smokers). Female participants had a median age of 53.9 (IQR 49.9, 59.9), a BMI of $25.6(23.6,27.8) \mathrm{kg} / \mathrm{m}^{2}$ and $431(13.0 \%)$ were ever smokers. Description of study population and median concentration of 14 subfractions is shown in Table 1.

We compared the sum of TG in the 14 subfractions to clinical chemistry measured total TG and found an increase of $0.34 \mathrm{mmol} / \mathrm{L}$ of NMR measured total TG for every $1 \mathrm{mmol} / \mathrm{L}$ increase in clinical chemistry measured total TG (Supplemental Figure 1). The overall study population distribution for the 14 TG subfractions was comparable across contributing studies, showed agreement between ethnicities in the Southall and Brent Revisited Study cohort (Supplemental Figure 2) and an overlap of TG measured in the fasting and non-fasting state (Supplemental Figure 3). Of the 14 TG subfractions, 12 had a skewed right tailed distribution, and two (medium and small HDL) had a more symmetrical distribution.

The reference intervals (2.5th-97.5th) percentile for 14 TG subfractions are shown in Table 2 and graphically in Figure 1. Wide reference intervals were observed in the VLDL subclass, for example the reference interval for TG in medium VLDL and small VLDL was $0.08-0.67 \mathrm{mmol} / \mathrm{L}$ and $0.10-0.46 \mathrm{mmol} / \mathrm{L}$, respectively. A smaller reference interval range was observed for TG in IDL, LDL and HDL subclass subfractions, for example, the reference interval range for TG in large HDL was $0.01-0.05 \mathrm{mmol} / \mathrm{L}$.

\section{Subgroup reference interval ranges}

Age and sex stratified reference interval ranges for 14 TG metabolites are presented in Supplemental Table 2. Reference interval ranges (2.5th-97.5th percentile) were comparable between male and female participants across metabolites. For example, among male participants aged $<55$ years, the large VLDL reference interval was in the range of $0.01-0.24 \mathrm{mmol} / \mathrm{L}$, and for female participants of the same age band, the reference interval range was $0.02-0.03 \mathrm{mmol} / \mathrm{L}$.

Figure 2 shows GAM curves and density distribution for the sum of VLDL, IDL, LDL and HDL subclass subfractions for age and BMI, and box plot for TG distribution by smoking status. Among male participants, TG concentration increased with age, with the most prominent age differences observed in the HDL subclass (Figure 2, left panel). By comparison, TG concentration differences were not as noticeable for female participants for whom concentrations were comparable for the VLDL, IDL, LDL and HDL subclasses by age. For both men and women, TG subfraction concentration increased with increasing BMI for the VLDL, IDL, LDL and HDL subclasses. Ever smokers had higher mean TG subfraction concentrations across all subclasses as compared to never smokers.

In the disease subgroups that were removed from all previous analyses reported above, the reference interval ranges for 14 subfractions were comparable between those with CVD $(\mathrm{N}=2719$ and those with T2DM $(\mathrm{N}=1325)$. In general, the largest variation in reference interval range across the subfractions between these subgroups was observed in the VLDL subclass (Supplemental Table 3). For example, the 2.5th to 97.5th reference interval range for $\mathrm{TG}$ in medium VLDL in CVD: $0.09,0.79 \mathrm{mmol} / \mathrm{L}$ and in T2DM: $0.08,0.84 \mathrm{mmol} / \mathrm{L}$.

The reference interval ranges across 14 TG subfractions were comparable in TG measured in the fasting and non-fasting state and were higher in the group with clinical chemistry measured total TG greater than $1.7 \mathrm{mmol} / \mathrm{L}$ as compared to less than $1.7 \mathrm{mmol} / \mathrm{L}$, see (Supplemental Table 3). 
Table I. Description of study sample.

\begin{tabular}{llr}
\hline & Male $(n=5574)$ & Female $(n=3299)$ \\
\hline Age, years & $61.7(52.0,67.6)$ & $53.9(49.9,59.9)$ \\
BMI, kg/m & $26.0(23.9,28.4)$ & $25.6(23.6,27.8)$ \\
Smoking, ever & $3027 / 5574(54.2)$ & $43 I / 3299(I 3.0)$ \\
Triglyceride subfraction (mmol/L) & & \\
Extremely large VLDL & $0.02(0.01-0.03)$ & $0.02(0.01-0.02)$ \\
Very large VLDL & $0.03(0.01-0.05)$ & $0.02(0.01-0.04)$ \\
Large VLDL & $0.10(0.06-0.18)$ & $0.09(0.05-0.16)$ \\
Medium VLDL & $0.23(0.16-0.35)$ & $0.24(0.16-0.34)$ \\
Small VLDL & $0.22(0.18-0.29)$ & $0.23(0.17-0.30)$ \\
Very small VLDL & $0.11(0.09-0.13)$ & $0.12(0.09-0.14)$ \\
IDL & $0.12(0.10-0.14)$ & $0.12(0.10-0.15)$ \\
Large LDL & $0.10(0.08-0.12)$ & $0.10(0.09-0.12)$ \\
Medium LDL & $0.05(0.04-0.06)$ & $0.05(0.04-0.05)$ \\
Small LDL & $0.03(0.02-0.04)$ & $0.03(0.02-0.04)$ \\
Very large HDL & $0.01(0.01-0.02)$ & $0.01(0.01-0.02)$ \\
Large HDL & $0.03(0.02-0.04)$ & $0.02(0.02-0.03)$ \\
Medium HDL & $0.05(0.04-0.06)$ & $0.05(0.04-0.06)$ \\
Small HDL & $0.05(0.04-0.06)$ & $0.04(0.04-0.05)$ \\
\hline
\end{tabular}

Note: Values are median (IQR) or \%.

VLDL: very-low density lipoprotein; IDL: intermediate-density lipoprotein; LDL: low-density lipoprotein; HDL: high-density lipoprotein

Table 2. Reference interval range of 14 TG-subfractions $(n=9073)$.

\begin{tabular}{lll}
\hline TG subfraction (mmol/L) & $2.50 \%$ & $97.50 \%$ \\
\hline Extremely large VLDL & 0.01 & 0.06 \\
Very large VLDL & $<0.01$ & 0.13 \\
Large VLDL & 0.01 & 0.42 \\
Medium VLDL & 0.08 & 0.67 \\
Small VLDL & 0.10 & 0.46 \\
Very small VLDL & 0.06 & 0.20 \\
IDL & 0.07 & 0.20 \\
Large LDL & 0.06 & 0.17 \\
Medium LDL & 0.02 & 0.08 \\
Small LDL & 0.01 & 0.05 \\
Very large HDL & $<0.01$ & 0.03 \\
Large HDL & 0.01 & 0.05 \\
Medium HDL & 0.02 & 0.08 \\
Small HDL & 0.03 & 0.08 \\
\hline
\end{tabular}

VLDL: very-low density lipoprotein; IDL: intermediate-density lipoprotein; LDL: low-density lipoprotein; HDL: high-density lipoprotein; TG: triglyceride.

\section{Discussion}

This study provides reference interval ranges for $\mathrm{TG}$ in 14 lipoprotein subfraction metabolites as measured by

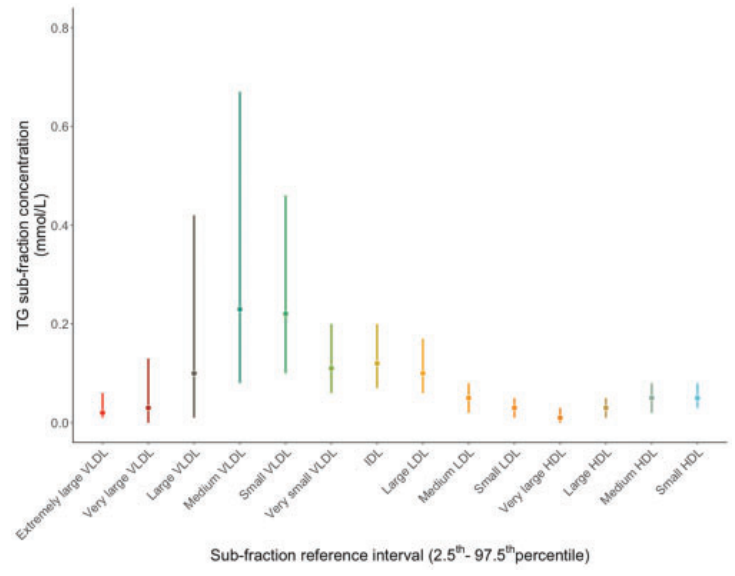

Figure I. Reference interval range for 14 triglyceride subfractions (median, 2.5th, 97.5th) percentiles.

Note. Please refer to the online version of the article to view the figure in colour.

NMR spectroscopy based on a sample of UK adults. There was agreement in the distribution of TG subfraction concentrations between ethnicities. TG concentrations for male and female participants increase with increasing age and BMI, are higher among ever 

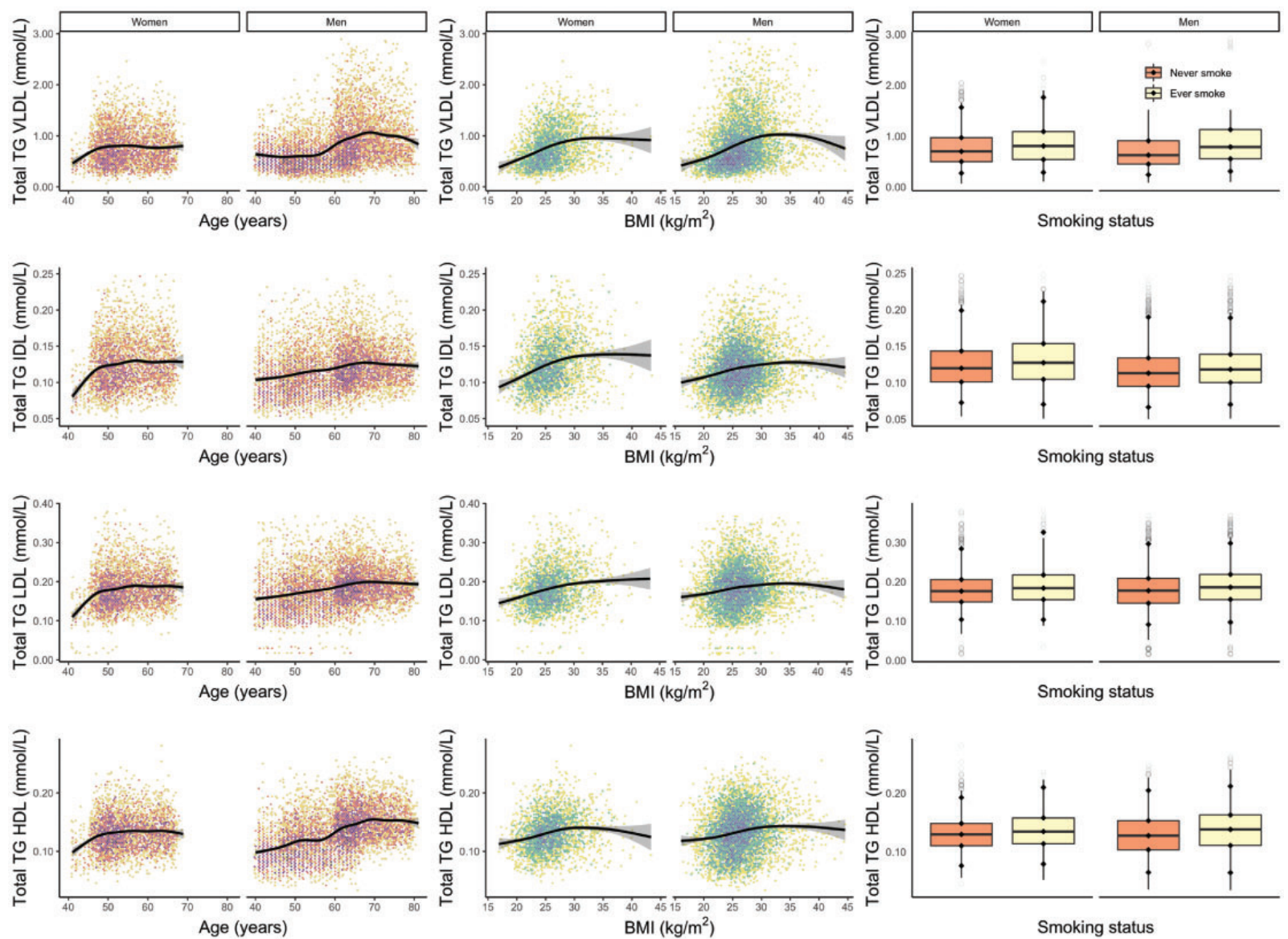

Figure 2. Distribution of TG concentration in VLDL, IDL, LDL and HDL subclass by age (left panel), body mass index (centre panel) and smoking status (right panel). N.b. Slope indicates a GAM estimate with $95 \%$ confidence interval. Tile colours represent the number of observations, with purple coloured tiles indicating a higher density. Smoking distribution (right panel) is based on data from BRHS, SABRE and WHII studies. All were significant at $P$ value threshold for $<0.00$. VLDL: very-low density lipoprotein; IDL: intermediate-density lipoprotein; LDL: low-density lipoprotein; HDL: high-density lipoprotein; BRHS: British Regional Heart Study; SABRE: the Southall and Brent Revisited Study; WHIl: the Whitehall II Study.

Note. Please refer to the online version of the article to view the figure in colour.

smokers and in those with CVD and T2DM as compared to disease-free subjects, and in individuals with total TG concentrations greater than $1.7 \mathrm{mmol} / \mathrm{L}$.

Lipid reference interval ranges are derived using clinical chemistry measurement of blood samples from a reference population and are necessary to enable clinicians to apply analytical data in healthcare delivery. For example, clinical chemistry estimates of LDL-C are measured in individuals and evaluated against an interval range to inform lifestyle or therapeutic intervention for CHD prevention. The role of TG in CHD risk is less clear. Meta-analysis from prospective observational studies has demonstrated higher concentrations of clinical chemistry measured total TG are associated with higher risk of CHD, but effect estimates attenuate to the null after adjustment for HDLC. ${ }^{17}$ On the other hand, Mendelian randomization studies support a potential causal association for
TG. ${ }^{18}$ The association of the major blood lipid fractions (LDL-C, TG and HDL-C) with CHD is seen across the whole of the concentration range, with no threshold value and we expect the same to be true of TG-containing lipoprotein subfractions. The reference ranges we report should not therefore be taken to imply that individuals whose measurements lie within these ranges are free of CHD risk. Rather we simply report the observed values in general UK populations.

NMR methodology offers the potential for more granular quantification of TG in different lipoproteins that would otherwise be unavailable using conventional approaches, enabling a more detailed investigation of TG-containing lipoprotein subfractions in relation to CHD risk and prognosis. Evidence from studies using this approach suggest CHD risk may be divergent depending on the type of lipoprotein subfraction. Two recent studies report observations of $\mathrm{TG}$ in 
VLDL subfractions may be more atherogenic and associated with a higher risk of CHD compared to TG in the IDL, LDL and HDL subclass subfractions. ${ }^{10,11}$

This study evaluates the concentration distribution and range of TG in 14 subfractions and includes data from multiple UK population cohorts and from male and female participants from a range of age groups and ethnicities including European, South Asian and African-Caribbean ancestry. We compare total TG measured using clinical chemistry and the sum of NMR TG across the 14 subfractions. Discrepancies between clinical chemistry methods and NMR measured total TG have been reported previously. ${ }^{10,17}$ In one such study, Balling et al. ${ }^{17}$ suggest differences in analytical calibration from measurement of TGs between the two methods may lead to measurement differences, with NMR quantification deemed as the more accurate method. ${ }^{18}$ TG concentrations are variable and, in addition to age, sex and ethnicity, can depend on factors such as food intake, fasting/nonfasting state, CVD and metabolic disorders such as T2DM..$^{19}$ Due to the relatively large sample sizes available, we observed a significant difference between the fasting and non-fasting distribution of subfractions. This significant difference did not prove relevant for determining the reference interval ranges, which were comparable to two decimal points. Moreover, it is postulated that the non-fasting state predominates the 24$\mathrm{h}$ cycle due to varying food intake patterns, and mean changes of $+0.3 \mathrm{mmol} / \mathrm{L}$ from baseline TG measures do not translate to clinically significant differences. Higher TG concentrations are observed in postmenopausal vs. premenopausal female. ${ }^{20}$ We report comparable TG concentrations across age groups; however, it is possible that a greater difference in TG concentration may be observed in a larger sample of women aged $>65$ than are included here. Due to the small numbers of current smokers in the available data across the contributing cohorts, we stratified by ever and never smoking status, instead of the more informative 'never', 'ex and 'current' smokers. We exclude participants with current CVD or T2DM in the main analyses; however, it is possible that the TG concentrations in the study population were altered by other diseases or by lipidlowering medication, which we were not able to account for in this study. It is likely TG monitoring is likely to occur in individuals at risk of, or with current CVD. Therefore, we provide additional reference intervals in participants with CVD and T2D.

We establish reference interval ranges of 14 TGcontaining lipoprotein subfractions for male and female by age, BMI, smoking status, CVD, T2DM and stratified by clinical chemistry measured total TG and fasting status for population-based cohorts from the UK population. Further studies would be needed to assess if the reference intervals presented here could be extended to a non-UK population and if the risks associated with the reference intervals identify a threshold within these ranges to inform CVD risk in a clinical setting. By doing so, the reference interval ranges may help to set realistic targets and guide research interests, contributing to the development of effective targeted TG-lowering therapies, aimed at, for example VLDL subfractions which may be the most atherogenic. ${ }^{10}$ NMR lipoprotein particle number and size have been assessed in relation to CHD; however, this study specifically presents reference range intervals for TG within the 14 subfractions. ${ }^{21}$ Further investigations would be needed to compare subfraction lipid composition, particle number concentration and size.

Metabolomics is becoming integrated with genomics to contribute to a better understanding of disease aetiologies and disease risk. ${ }^{22}$ It is likely that quantitative metabolomics will be incorporated into large biobanks, which would extend the relevance of sample collection and encourage the life-long assessment of metabolic health. ${ }^{6}$ TG subfraction reference interval ranges may help complement current routine clinical chemistry measures of lipids and become an integral tool in targeted patient management and improved disease risk prediction and prevention.

\section{Conclusion}

This study is the first to establish reference interval ranges for 14 TG-containing lipoprotein subfraction metabolites, measured using the Nightingale NMR platform for men and women in a UK population. NMR measures of lipoproteins may provide insights into biological processes compared to clinical chemistry measures of TG and lead to greater insights for the role of TG in CVD, emphasizing the importance of appropriate reference interval ranges for future clinical decision making.

\section{Acknowledgements}

The authors acknowledge the invaluable discussions with members of their laboratories and colleagues. RJ is a PhD candidate at UCL funded by the BHF.

\section{Declaration of conflicting interests}

The author(s) disclosed receipt of the following financial support for the research, authorship and/or publication of this article: JP has received grant funding from GSK to conduct methodological research on Electronic health records to improve the drug discovery process. DAL has received support from Medtronics Ltd and Roche Diagnostics for biomarker research not related to this study.

\section{Funding}

The author(s) disclosed receipt of the following financial support for the research, authorship, and/or publication of this article: AFS is supported by BHF grant PG/18/5033837 and the UCL BHF Research Accelerator AA/18/6/ 34223. TG and DAL work in a Unit that is supported by the University of 
Bristol and UK Medical Research Council (MC_UU_00011/4 and MC_UU_00011/6).

This work was supported by the British Regional Heart Study team for data collection. The British Regional Heart study is supported by British Heart Foundation grant (RG/13/16/30528). The British Heart Foundation had no role in the design and conduct of the study; collection, management, analysis, and interpretation of the data; preparation, review, or approval of the manuscript; and decision to submit the manuscript for publication.

The SABRE study was funded at baseline by the Medical Research Council, Diabetes UK, and British Heart Foundation and at follow-up by the Wellcome Trust $(082464 / \mathrm{Z} / 07 / \mathrm{Z})$, British Heart Foundation (SP/07/001/ 23603, PG/08/103, PG/12/29/29497 and CS/13/1/30327) and Diabetes UK(13/ 0004774) NC and ADH receive support from the National Institute for Health Research University College London Hospitals Biomedical Research Centre.

The Whitehall II Study has been supported by grants from the UK Medical Research Council (MRC K013351, R024227, S011676); the British Heart Foundation (PG/11/63/29011 and RG/13/2/30098); the British Health and Safety Executive; the British Department of Health; the National Heart, Lung, and Blood Institute (R01HL036310); the National Institute on Ageing, National Institute of Health (NIA R01AG056477, R01AG034454); the Economic and Social Research Council (ES/J023299/1). M Kivimaki is supported by the MRC, UK (K013351, R024227, S011676), NIA, US (R01AG056477) and the Academy of Finland (311492).

\section{Ethical approval}

Local research ethics committees provided ethical approval and participants gave written informed consent.

\section{Guarantor}

RJ.

\section{Contributorship}

RJ, GW, ADH and AFS contributed to the idea and design of the study. RJ drafted the initial the manuscript, GW ADH and AFS revised the manuscript. RJ performed the presented analyses, had complete access to all the data, and takes responsibility for the integrity and accuracy of the presented results. JE, TG, DAL, JP, OP, TS, TT, AH, NC, MK, DK, MK, ADH, JPC, SH, ADH and AFS contributed to data acquisition and interpretation. All authors reviewed and edited the manuscript and approved the final version of the manuscript.

\section{ORCID iD}

Roshni Joshi (DD https://orcid.org/0000-0002-0236-572X

\section{Supplemental material}

Supplemental material for this article is available online.

\section{References}

1. Nordestgaard BG and Varbo A. Triglycerides and cardiovascular disease. Lancet 2014; 384: 626-635.

2. Toth PP. Triglyceride-rich lipoproteins as a causal factor for cardiovascular disease. Vasc Health Risk Manag 2016; 12: 171-183.
3. Hegele RA, Ginsberg HN, Chapman MJ, et al. The polygenic nature of hypertriglyceridaemia: implications for definition, diagnosis, and management. Lancet Diab Endocrinol 2014; 2: 655-666.

4. Miller M, Stone NJ, Ballantyne C, et al. Triglycerides and cardiovascular disease. Circulation 2011; 123: 2292-2333.

5. Pencina MJ, D'Agostino RB, Larson MG, et al. Predicting the 30-year risk of cardiovascular disease: the Framingham heart study. Circulation 2009; 119: 3078-3084.

6. Soininen P, Kangas AJ, Würtz P, et al. Quantitative serum nuclear magnetic resonance metabolomics in cardiovascular epidemiology and genetics. Circ Cardiovasc Genet 2015; 8: 192-206.

7. Ala-Korpela M, Kangas AJ and Soininen P. Quantitative high-throughput metabolomics: a new era in epidemiology and genetics. Genome Med 2012; 4: 36 .

8. Lifelong health belongs to everyone, https://nightingalehealth.com/ (accessed 26 September 2020).

9. Würtz P, Kangas AJ, Soininen P, et al. Quantitative serum nuclear magnetic resonance metabolomics in large-scale epidemiology: a primer onomic technologies. Am J Epidemiol 2017; 186: 1084-1096.

10. Holmes MV, Millwood IY, Kartsonaki C, et al. Lipids, lipoproteins, and metabolites and risk of myocardial infarction and stroke. J Am Coll Cardiol 2018; 71: 620-32.

11. Joshi R, Wannamethee SG, Engmanna J, et al. Triglyceride-containing lipoprotein sub-fractions and risk of coronary heart disease and stroke: a prospective analysis in 11,560 adults. Eur J Prev Cardiol 2020; 27: 16171626.

12. Shah T, Engmann J, Dale C, et al. Population genomics of cardiometabolic traits: design of the University College London-London School of Hygiene and Tropical Medicine-Edinburgh-Bristol (UCLEB) Consortium. PLoS One 2013; 8: e71345.

13. Wurtz P, Tiainen M, Makinen V-P, et al. Circulating metabolite predictors of glycemia in middle-aged men and women. Diabetes Care 2012; 35: 1749-1756.

14. Würtz P, Soininen P, Kangas AJ, et al. Characterization of systemic metabolic phenotypes associated with subclinical atherosclerosis. Mol Biosyst 2011; 7: 385-393.

15. Würtz $P$, Havulinna AS, Soininen $P$, et al. Metabolite profiling and cardiovascular event risk. Circulation 2015; 131: 774-785.

16. Soininen $\mathrm{P}$, Kangas AJ, Würtz $\mathrm{P}$, et al. High-throughput serum NMR metabonomics for cost-effective holistic studies on systemic metabolism. Analyst 2009; 134: 1781.

17. Balling M, Langsted A, Afzal S, et al. A third of nonfasting plasma cholesterol is in remnant lipoproteins: lipoprotein subclass profiling in 9293 individuals. Atherosclerosis 2019; 286: 97-104.

18. Holmes MV and Ala-Korpela M. What is 'LDL cholesterol'? Nat Rev Cardiol 2019; 16: 197-198.

19. Brunzell JD. Hypertriglyceridemia. N Engl J Med 2007; 357: 1009-1017.

20. Anagnostis P, Bitzer J, Cano A, et al. Menopause symptom management in women with dyslipidemias: an EMAS clinical guide. Maturitas 2020; 135: 82-88.

21. El Harchaoui K, van der Steeg WA, Stroes ESG, et al. Value of lowdensity lipoprotein particle number and size as predictors of coronary artery disease in apparently healthy men and women. The EPIC-Norfolk prospective population study. J Am Coll Cardiol 2007; 49: 547-553.

22. Shah SH and Newgard CB. Integrated metabolomics and genomics. Circ Cardiovasc Genet 2015; 8: 410-419. 